\title{
Simulation-based Learning of Ultrasound-guided Techniques
}

\section{EL Bouazzaoui Abderrahim*, Boukatta Brahim, Touzani Soumaya, Tsigbe Wisdom Kwasi, Houari Nawfal and Kanjaa Nabil}

Intensive Care Unit, Hassan II University Hospital and Medical School of Fez, Sidi

Mohammed Benabdellah University, Fez, Morocco

*Corresponding Author: EL Bouazzaoui Abderrahim, Intensive Care Unit, Hassan II University Hospital and Medical School of Fez, Sidi Mohammed

Benabdellah University, Fez, Morocco.
Received: September 03, 2021

Published: November 17, 2021

(C) All rights are reserved by EL Bouazzaoui Abderrahim., et al.

\section{Abstract}

In the field of intensive care, anesthesia and emergency medicine, performing invasive acts using Ultrasound guidance such as vascular puncture or performing locoregional anesthesia techniques is not devoid of iatrogenicity and adverse events which can sometimes be serious and fatal. Learning by simulation is an essential step aimed at reducing the risk of these serious undesirable events occurring when performing these techniques. The learning models offered by manufacturers remain demanding. We offer a simple and less demanding model on how to visualize the needle and to follow its progression during the realization of the invasive techniques under Ultrasound guidance. In our context, our resident anesthetists undergoing training benefit from the contribution of this model during their first training courses by simulating these invasive techniques. This allows these trainees to learn the concepts of in-plane and out-of-plane puncture of ultrasound as well as to master the techniques for tracking puncture needle progression. These simulation models would make the learning process of these invasive acts quick and easy while reducing the risk of iatrogenic incidents occurring.

Keywords: Simulation; Learning; Ultrasound-guided Techniques

\section{Introduction}

The performance of Anaesthesia techniques (venipuncture, arterial puncture, regional anaesthesia) are frequent acts not deprived of iatrogenic infections which could sometimes be catastrophic. Currently, Learned Societies recommend the use of Ultrasound guidance when performing these acts. The Ultrasound Machine has become the new "tool box" of the Anesthesiologist. The learning process of these new techniques is key to assess on a step-by-step basis. Simulation- based learning is an essential step that facilitates the learning of how to perform Invasive Acts. We are proposing simple, less onerous physical model on how to learn to visualize the needle and follow its progression during Ultrasound explorations. In fact, in the instance where there exists a risk of tissue lesion during the learning process of mastering needle guid- ance skill, the learning process must be realized in an ideal context. There are different kinds of models which facilitate the learning process of needle guidance before proceeding to operate in a Clinical setting. Whichever Model you choose, it must ensure the acquisition of skills and enhance the assimilation of basic knowledge and then advanced knowledge in Imagery. The transfer of knowledge from a model simulating a real context to a clinical setting requires the successful integration of skills acquired from Ultrasound on one hand and the needle guidance skill on another all in one coherent manner.

\section{Materials and Methods}

- An ultrasound Machine with different types of probes.

- $\quad$ Echogenic needles of different sizes. 
- A physical model has been designed to be used for the demonstration; it's a cylindrical object of Length $25 \mathrm{~cm}$ normally used as the cover of the Ultrasound probe to ensure asepsis of the probe during the performance of aseptic procedures (Figures 1 and 2). One end of the Cylinder is hermetically attached to the base of a plastic bottle, the other end of the cylinder is attached to the Cephalic part of the aforementioned plastic bottle consisting of a cork. This cork allows the cylinder to be filled with transonic gel which is used with the Ultrasound. A vascular prosthesis (biomedical) recovered at the end of a vascular surgery procedure performed in our Surgical Theatre is passed across the inside length of the Cylinder. After filling the arterial prosthesis with water coloured by a red tint, both ends of the arterial prosthesis were clamped. The second model used was fresh turkey thigh provided by the trainee doctors on the day of the simulation (Figure 3).

The second part of this simulation-based training was scheduled for the second day. It was dedicated to the learning of human anatomy sounds with the help of a mannequin (Anesthesia Residents). It essentially had to do with sonoanatomy of the cervical region \{Central venous line, cervical plexus block (interscalene block, supra or infra clavicular block), axillary region, Femoral\} and finally sonoanatomy of the thoraco-abdominal wall (Thoracic paravertebral block, TAP block).

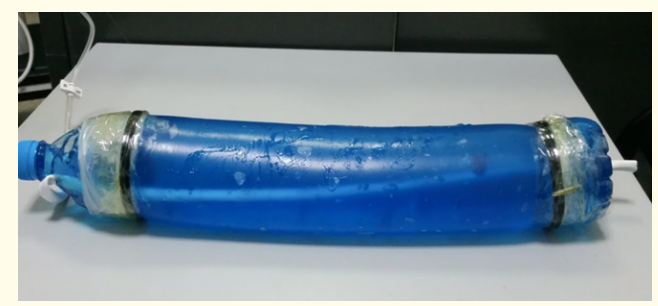

Figure 1: The physical model: A $25 \mathrm{~cm}$ long plastic cylinder with one end hermetically sealed to the base of a plastic bottle. The other end of this cylinder is attached to the cephalic part of the plastic bottle with the stopper. This cap allows the cylinder to be filled with ultrasound gel. A vascular prosthesis (biomedical) recovered at the end of a vascular surgery procedure performed in our Surgical Theatre is passed across the inside length of the Cylinder. After filling the arterial prosthesis with water coloured by a red tint, both ends of the arterial prosthesis were clamped.

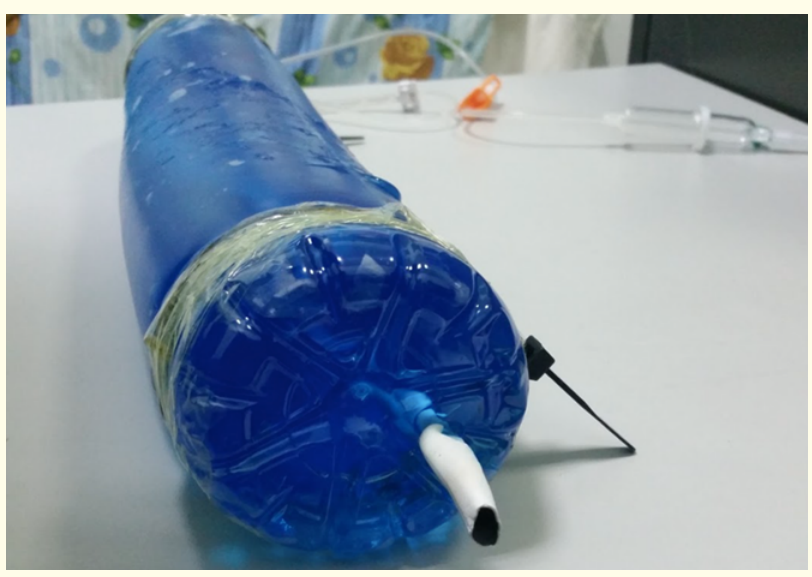

Figure 2: A vascular prosthesis clamped on both sides and filled with a tinted liquid crosses the cylinder lengthwise. The arterial prosthesis is filled by a red tinted liquid. This prosthesis is easy to puncture with a needle.
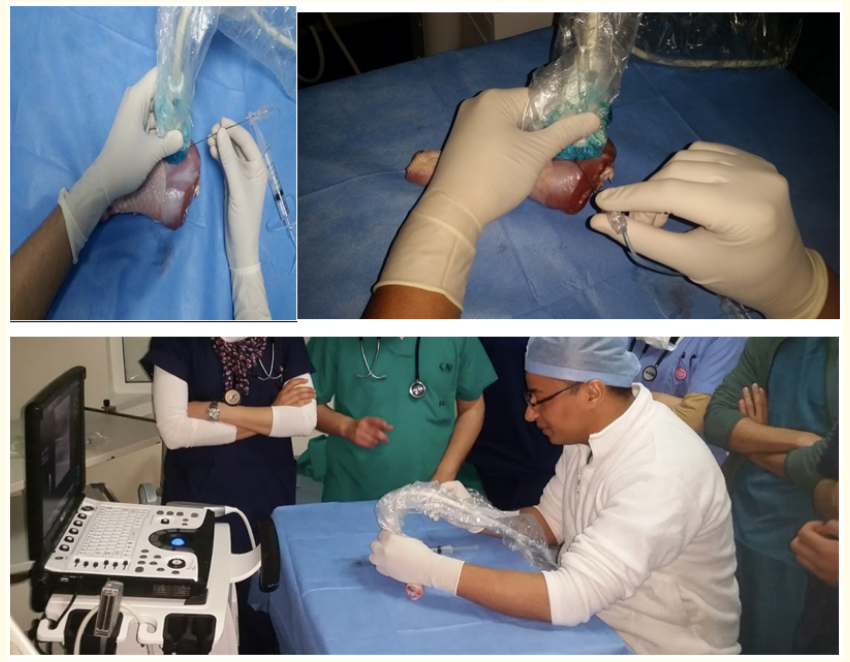

Figure 3: The animal model. Regional Anesthesia technique learning model "turkey thigh". Trainees learn the concepts of in-plane and out-of-plane ultrasound puncture as well as mastering the techniques of tracking the progress of the puncture needle during simulated invasive procedures. 

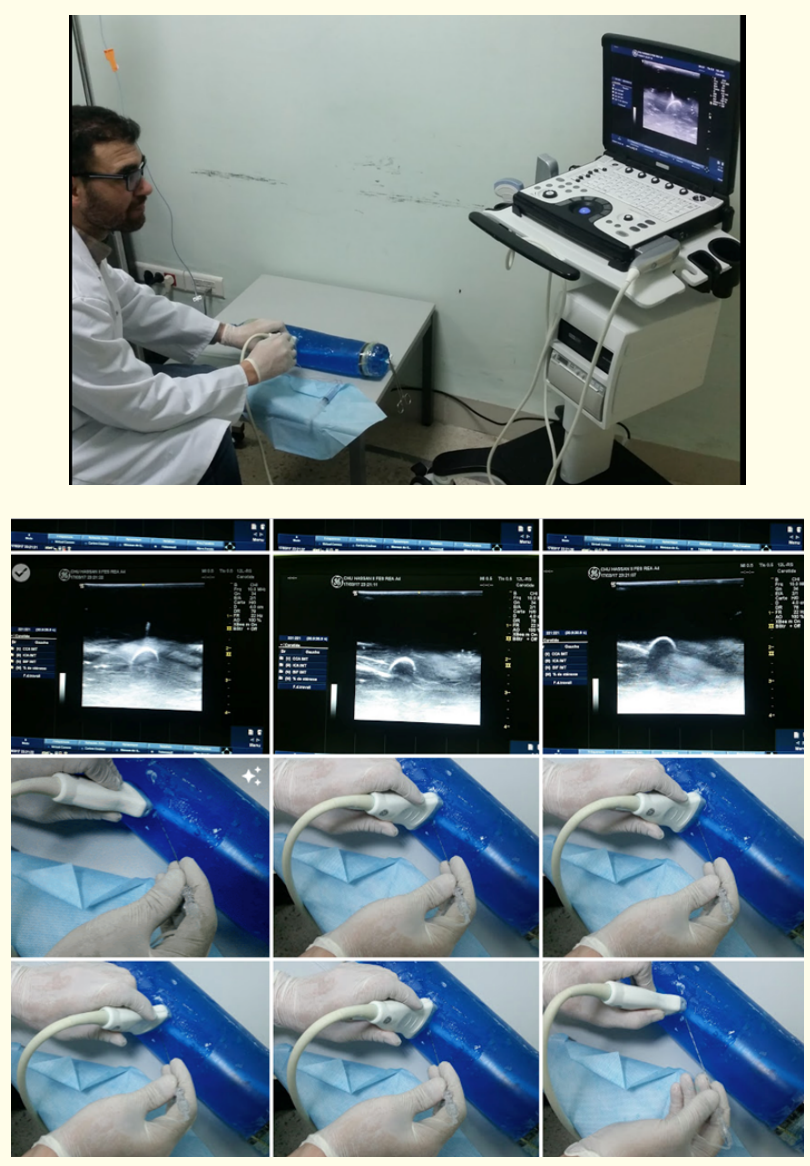

Figure 4: Trainees learn the concepts of in-plane and out-of-plane ultrasound puncture as well as mastering the techniques of tracking the progress of the puncture needle during simulated invasive procedures.

\section{Results}

By virtue of these learning models, the trainee doctors understood the concept; out-of-plane and in-plane of ultrasounds (Figure 4). This allowed the trainees to introduce an echogenic needle and to follow its progression in the ultrasound field as well as to target "the vessel to puncture" represented in the physical model by the vascular prosthesis. An initial scanning allows you to view the vessels of the model on small axis and large axis view. In maintaining the probe in a way to achieve a view in small axis for an outof-plane approach (OOP) of the vascular access route, or performing a 90 degrees rotation to obtain a view in large axis in the case of in-plane approach, It then becomes possible to achieve the vascular catheter placement. The "turkey thigh model" has enabled us to obtain a reliable human sonoanatomy similar to abdominal wall sonoanatomy, as a result of the interposition of different muscles of the turkey thigh (Figure 5).

\section{Discussion}

The use of Ultrasound guidance for the placement of Central Venous Line or for Regional Anaesthesia acts is on the rise. Specific and Technical Training are increasingly demanded by different practitioners; However, these Ultrasound techniques require learning at three levels:

\section{- Recognition of the main Ultrasound elements.}

- Handling of the probe in order to obtain quality images.

- Acquiring adequate skill on how to align the needle and the probe at all times in order to clearly move the needle to the choosen target (blood vessel or nerve).

It is essential to identify the structures that must be avoided which are at risk to be compromised that is either pierced or damaged (e.g. the first rib, the pleura in the supraclavicular region). It is due to this reason that it is advisable to practice regularly with both hands, and to experiment with both types of in-plane and out-ofplane approaches. In order to acquire skills needed for needle guidance which is necessary for both vascular access approach and Ultrasound-guided regional anaesthesia, easy-to-construct physical models were invented. There are two published models that can be used for training; The first uses a parallelepipedal gelatin phantom crossed by a tube [1]. Although it provides technical parallels to needle guidance in tissues, these models are unable to demonstrate the dynamic real-time perception of injected fluids nor the soft tissue movements that contribute to real clinical setting, for this reason a second model was invented using a piece of Turkey breast in which an olive is placed [2]. When needle guidance techniques are mastered in the context of the model, caution is required in transitioning to clinical application. Clinical monitoring of paresthesia, possible resistance or pain on injection, careful suction and visual confirmation of the location of the needle and the diffusion of local anesthetics over time by the injection will improve the results of the procedure and minimize the risk to patients. When the skill requiring the keeping of the needle reliably in the plane (in -plane) 
over the entire path between the surface and the target using both hands is acquired, the model can be used progressively to perform more difficult gestures. We can then focus on targets of smaller diameters; puncture of the "vascular prosthesis" presented by the physical model or even target a very specific object in the inside of the turkey thigh such as a tendon or an intermuscular aponeurosis. With the in-plane approach, the target is centered on the screen and the needle path is planned. It should enter the field of view from the upper left or upper right corner. The needle is inserted below the major axis of the probe and maneuvered in line with the probe. The advantage of the in-plane approach is that it provides a view of the entire needle and its tip when the probe and needle are properly aligned. This is especially important in the presence of vital structures near the target or in the intended path of the needle (e.g. supraclavicular block is performed near the pleural dome). It also shows the distance between the tip of the needle and the target. The inconvenience to the In-plane approach is that it is more difficult because it requires precise alignment of the Ultrasound probe with the needle and the target. The needle path is not the shortest distance between the skin and the target; this often has to penetrate the muscle layers, which makes this technique more painful. On the other hand, the out-of-plane approach(OOP) does not allow continuous visualization of the needle as it passes through the tissues, but only when it passes through the thin anatomical plane of the probe's beam shown on the screen. The out-of-plane technique usually does not indicate the relationship of the needle to anatomical structures before or after it has passed through the bundle. Ultrasound techniques for vascular access routes or local anaesthesia is part of the training for Anesthesiologists. The clinical expertise is developed from the performance of series of procedures. There is a learning curve for all techniques that involves the completion of a given number of blocks, indeed, T. DESSIEUX., et al. all evaluated the learning curve using a mannequin in 12 Anaesthesia residents new to Ultrasound during an Ultrasound guided Regional Anaesthesia workshop. According to this study, four trials were required to properly set up the Ultrasound Machine, locate a target and hydrolocate it. Ultrasound guided Regional Anaesthesia Procedures appear to be a rapid learning technique, using this type of workshop [3]. SITES., et al. followed the evolution of the learning curve (process) of Ultrasound guided Regional Anaesthesia acts in 6 Residents evaluated over a period of one month; thus allowing them to improve the realization time of the act and the precision during the period of time of the study (Figure 6). Likewise, B-V NGUYEN., et al.
Demonstrated that after an average of 8 procedures the technique of placing central venous line (jugular vein access route) using the Ultrasound seemed to be acquired by novices and the total procedure time became very short (Figure 7) [4,5].

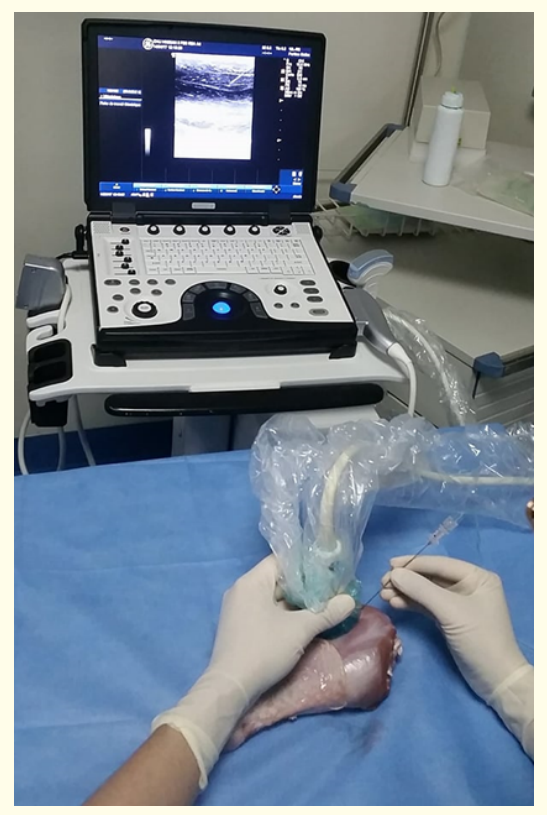

Figure 6: The turkey thigh model makes it possible to reproduce Ultrasound images close to those found in clinical settings, for example, when performing anesthetic blocks of the abdominal wall.

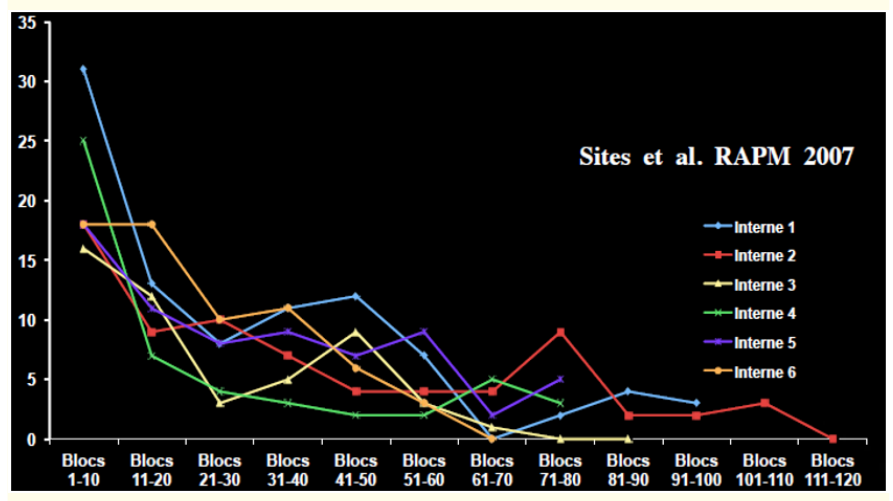

Figure 7: learning curve of 6 residents over a period of one month. 


\section{Conclusion}

Learning Ultrasound guidance techniques for Vascular Access Routes or Regional Anaesthesia procedures is part of the training for Anesthesiologists. Several models for learning these techniques through simulation are available. The evaluation of the practices is essential, it should be based on how to obtain the best image, on a rigorous training of the guidance of the needle and on the analysis of the optimal distribution of the local anesthetic agent. The evolution of the use of Ultrasound is promising and a highly recommendable prospect that makes the Ultrasound technique more and more accessible for use.

\section{Bibliography}

1. Nicholson RA and Crofton M. "Training phantom for ultrasound guided biopsy". British Journal of Radiology 70 (1997): 192-194.

2. Sites BD., et al. "The learning curve associated with a simulated ultrasound-guided interventional task by inexperienced anesthesia residents". Regional Anesthesia and Pain Medicine 29 (2004): 544-548.

3. T Dessieux., et al. "Évaluation de la courbe d'apprentissage des internes pour l'échoguidage sur un fantôme". Annales Françaises D'anesthésie Et De Réanimation 27.10 (2008) 797-801.

4. Sites., et al. RAPM (2007).

5. BV Nguyen. "Courbe d'apprentissage de la pose de voie veineuse centrale jugulaire sous échoguidage”. Réanimation 21 (2012): S275-S278.

Volume 5 Issue 12 December 2021

(C) All rights are reserved by EL Bouazzaoui Abderrahim., et al. 